\title{
REDES SOCIAIS E EMPREENDEDORISMO EM BIOTECNOLOGIA.

\author{
O PROCESSO DE AGLOMERAÇÃO EM TORNO DE NÚCLEOS \\ DE PRODUÇÃO DE CONHECIMENTO
}

\author{
MARGARIDA Fontes ${ }^{1}$ \\ CRISTINA DE SOUSA ${ }^{1}$ \\ PEDRO VIDEIRA ${ }^{2}$
}

\begin{abstract}
Resumo - Este $\operatorname{artigo~}^{3}$ aborda a aglomeração de novas empresas de biotecnologia em torno de núcleos de produção de conhecimento, centrando-se no papel das redes sociais no acesso ao conhecimento científico e tecnológico. A abordagem toma em consideração o impacto de várias formas de proximidade - geográfica, social, cognitiva e organizacional - no desenvolvimento de relações chave e na sua utilização para obter conhecimento científico e tecnológico. Tal permite aferir a importância relativa de fontes de conhecimento próximas e distantes e explicar as decisões tomadas pelos empreendedores em termos de mobilização das redes que lhes dão acesso. Na exploração empírica recorre-se a uma metodologia para reconstruir as redes sociais e medir os diferentes tipos de proximidade.
\end{abstract}

Palavras-chave: Redes sociais, empresas baseadas na ciência, acesso ao conhecimento, proximidade, biotecnologia.

\begin{abstract}
SocIAL NETWORKS AND ENTREPRENEURSHIP IN BIOTECHNOLOGY. THE CLUSTERING OF FIRMS AROUND REGIONAL CENTERS OF KNOWLEDGE PRODUCTION. This paper ${ }^{4}$ addresses the clustering of new biotechnology firms around centres of knowledge production, by focusing on the role played by social networks in terms of access to scientific and technological knowledge. Our approach takes into consideration the impact of various forms of proximity - geographical, social, cognitive and organisational - upon the development of key relationships, as well as upon their use for knowledge acquisition. It enables us to assess the relative importance of local and distant knowledge sources, and to interpret the decisions taken by entrepreneurs with respect to network mobilisation. The empirical operationalisation of this approach is then discussed based on a suggested methodology that makes it possible to reconstruct social networks and to measure different types of proximity.
\end{abstract}

Recebido: 30/10/2008. Revisto: 22/01/2009. Aceite: 30/01/2009.

1 INETI/DMS e DINAMIA E-mails: margarida.fontes@ineti.pt; cristina.sousa@ineti.pt

2 DINAMIA, ISCTE. E-mails: p videira@yahoo.com

3 Investigação conduzida no âmbito do projecto ENTSOCNET "Redes sociais, empreendedores e acesso ao conhecimento", financiado pela Fundação para a Ciência e Tecnologia (POCI/ $\mathrm{ESC} / 60500 / 2004)$. 
Key words: Social networks, science-based firms, knowledge access, proximity, biotechnology.

Résumé - LES RÉSEAUX SOCIAUX ET LES ENTREPRISES DE BIOTECHNOLOGIE: LEUR ATTRACTION PAR LES CENTRES DE PRODUCTION DE CONNAISSANCE. On aborde le problème de l'implantation des nouvelles entreprises de biotechnologie près des centres de production de connaissance, en étudiant le rôle joué par les réseaux sociaux dans l'accès aux connaissances scientifiques et technologiques. On considère l'impact des diverses formes de proximité, géographique, sociale, cognitive et organisationnelle, dans la création des relations qui ouvrent l'accès aux connaissances scientifiques et technologiques nécessaires. On apprécie l'importance relative des sources de connaissance, proches ou distantes, et on explique les décisions prises par les entrepreneurs selon les réseaux d'accès qu'ils ont mobilisés. Pour concrétiser ce point de vue, on présente une méthodologie, permettant la reconstruction de réseaux sociaux et l'évaluation des divers types de proximité.

Mots-clés: Réseaux sociaux, entreprise de base scientifique, accès à la connaissance, proximité, biotechnologie.

\section{INTRODUÇÃO}

A literatura sobre a evolução do sector da biotecnologia em várias regiões do mundo (Boston, São Francisco, Cambridge, Jena) salienta o papel desempenhado por diversas organizações em diferentes níveis espaciais. Verifica-se que as empresas de biotecnologia tendem a aglomerar-se em torno de centros de investigação, de "star scientists", de clientes importantes ou, no caso dos EUA, de organizações de capital de risco (Audretsch e Stephan, 1996; Cooke, 2006; Powell et al., 2002, Zucker et al., 1998). Mas, por outro lado, as empresas neste sector estão frequentemente muito internacionalizadas, estabelecendo relações do mais variado tipo com organizações distantes (Owen-Smith e Powell, 2004).

Este trabalho aborda o papel das redes sociais no acesso ao conhecimento científico e tecnológico pelas novas empresas portuguesas de biotecnologia, centrando-se nas relações entre empresas e centros de investigação. De forma mais concreta, analisa-se a aglomeração das empresas em torno de núcleos de produção de conhecimento científico e tecnológico e procura-se compreender o significado dessa localização nas suas estratégias de aquisição de conhecimento. Ou seja, pretende avaliar-se o papel desempenhado pelas redes efectivamente estabelecidas com tais núcleos, comparativamente com o desempenhado por redes organizadas com fontes de conhecimento científico e tecnológico mais distantes.

O artigo está organizado da seguinte forma: na próxima secção é revista a literatura relevante, abordando-se a natureza do empreendedorismo em biotecnologia, o papel das redes sociais no empreendedorismo e os debates sobre a 
proximidade. A terceira secção é dedicada ao levantamento sobre o modo como as redes sociais facilitam o acesso ao conhecimento, através da geração de várias formas de proximidade, que podem favorecer (ou não) a aglomeração em torno dos centros que o produzem. A quarta secção é dedicada à investigação empírica destas questões para o caso das empresas portuguesas de biotecnologia. Faz-se uma breve apresentação do sector e retiram-se algumas ilações para a investigação a realizar. De seguida propõe-se uma abordagem metodológica ao problema em dois passos: (re)construção das redes sociais mobilizadas no acesso ao conhecimento; análise de proximidade relativamente aos centros de investigação e avaliação das condições oferecidas pelo contexto local/regional a esse nível.

\section{EMPREENDEDORISMO, PROXIMIDADE E REDES SOCIAIS}

\section{Caracterização do empreendedorismo em biotecnologia}

O empreendedor é alguém que explora uma oportunidade que outros não tinham percepcionado, propondo-se fazer algo novo e que portanto envolve risco e incerteza. O processo de empreendedorismo requer a confluência de dois fenómenos: a existência de oportunidades lucrativas e a presença de indivíduos empreendedores (Shane e Venkataraman, 2000). A exploração da oportunidade exige a obtenção de um conjunto de recursos, cuja natureza depende da oportunidade percepcionada (Johannisson, 1998). A formação de uma nova empresa é um processo complexo e dinâmico, onde intervêm factores de natureza muito distinta (económicos, sociais, culturais): o empreendedorismo é um processo de aprendizagem que envolve a assimilação e a troca de informação com o ambiente.

O empreendedorismo em biotecnologia apresenta algumas especificidades, associadas ao facto de este ser um sector onde a proximidade entre a ciência e o mercado cria oportunidades ao nível da transformação dos resultados da investigação científica em tecnologias, produtos e serviços (Zucker et al., 1998). Dado que, nesta área, o conhecimento científico e tecnológico evolui e se difunde de forma rápida, é indispensável procurar constantemente conhecimento novo, absorvê-lo e transformá-lo em capacidades internas da empresa (Witt e Zellner, 2007). Assim, o acesso à investigação realizada no exterior da empresa e, nomeadamente, a novo conhecimento científico, pode assumir um papel decisivo (Liebeskind et al., 1996). Ora as novas descobertas científicas beneficiam frequentemente de uma certa "exclusão natural" (Zucker et al., 1998), que torna a interacção com os cientistas envolvidos na sua produção indispensável nos processos de transferência. Daí que empresas criadas por empreendedores científicos tenham vantagens na identificação e exploração das novas oportunidades e que o desempenho dessas empresas seja positivamente afectado pela existência de laços com centros de investigação de relevo (Murray, 2004). Dada a variedade de domínios que contribuem para a biotecnologia e a natureza distribuída da 
produção de conhecimento, pode tornar-se necessário recorrer a uma variedade de organizações, dispersas por diferentes localizações (Powell et al., 1996).

Embora o conhecimento científico e tecnológico surja como um recurso crítico, a sua transformação em tecnologias, produtos ou serviços e a introdução destes no mercado, requer outros recursos e competências que os empreendedores científicos frequentemente não possuem e que podem ter algumas dificuldades em mobilizar, devido ao seu background e à natureza das organizações de onde provêm (Ensley e Hmieleski, 2005). Entre estes avulta o acesso ao capital, bem como a um conjunto de activos complementares associados à produção $\mathrm{e}$ comercialização de produtos e serviços que podem ser altamente inovadores (Pisano, 1991). Conquanto o foco desta investigação esteja na aquisição de conhecimento científico e tecnológico, deve ser tido em consideração que a obtenção de um leque amplo de outros recursos, de acesso mais ou menos complexo, poderá ter uma forte influência nas decisões estratégicas das novas empresas de biotecnologia, nomeadamente em termos de localização (Stuart e Sorenson, 2003).

Dada a necessidade premente de aceder a fontes externas de conhecimento científico e tecnológico - sobretudo numa fase inicial, em que a base de conhecimento da empresa está em formação, dependendo muito de competências detidas ou acedidas pelos empreendedores - as redes sociais surgem como um elemento crítico para as novas empresas de biotecnologia.

\section{Redes sociais e sua importância no empreendedorismo}

\subsection{Redes sociais e empreendedorismo}

Nas últimas décadas tem-se assistido a uma evolução na literatura sobre as origens do empreendedorismo: de uma abordagem centrada nos traços de personalidade, factores psicológicos e variáveis demográficas, tem-se passado para uma abordagem que encara o empreendedorismo como processo socio-económico (Granovetter, 1985) integrado em estruturas de rede (Aldrich e Zimmer, 1986; Johannison, 1988; Carsrud e Johnson, 1989; Uzzi, 1997), abandonando-se a tradicional visão do empreendedor como um indivíduo isolado. Nesta perspectiva, o capital social e as redes sociais assumem um papel de relevo no empreendedorismo, considerando-se que a formação e o desenvolvimento da empresa são facilitados (ou condicionados) pelas redes sociais dos seus fundadores (redes pessoais) e pelo contexto social em que a empresa está inserida (redes interorganizacionais). Estas redes permitem contornar algumas das restrições que o empreendedor enfrenta no processo de criação, facilitando a obtenção de recursos na sua envolvente.

$\mathrm{Na}$ análise da importância das redes sociais no processo de empreendedorismo, tem sido dada particular atenção às novas empresas de base tecnológica, onde geralmente o conhecimento científico e tecnológico se encontra na génese 
da oportunidade e na base da vantagem competitiva. Como a aquisição e a exploração de conhecimento são processos sociais (Kogut e Zander, 1992), argumenta-se que, através da interacção social, é possível aumentar a profundidade, amplitude e eficiência das trocas de conhecimento (Lane e Lubatkin, 1998). Por outro lado, quando a empresa não tem reputação estabelecida ou quando o valor das suas tecnologias não está provado, a rede social pode fornecer credibilidade (Powell et al., 1996).

\subsection{Propriedades das redes sociais}

Uma rede social (RS) é composta por diversos agentes individuais (os nós, ou actores da rede) ligados por relações sociais (os elos, ou laços) e pelos mecanismos de regulação dessas interacções (Castilla et al., 2000). É comum distinguir os laços directos (relação directa entre dois actores) dos laços indirectos (ligação de dois nós por intermédio de outros nós).

A literatura das RS distingue entre laços fortes e fracos, sendo os primeiros caracterizados por maiores níveis de proximidade social e reciprocidade. De acordo com Granovetter (1973), a força dos laços deve ser analisada através de uma combinação entre a frequência e duração, intensidade emocional, intimidade e reciprocidade que os caracterizam. O desenvolvimento de laços fortes requer esforços concretos e uma interacção regular, pelo que é geralmente favorecido pela proximidade física dos actores. Na literatura tal proximidade surge associada à frequência da interacção face-a-face (McEvily e Zaheer, 1999), à ocorrência de contactos não planeados (Fornahl, 2005) e também à confiança (Bönte, 2008; Johanisson, 1998). No entanto, a existência de muitos laços deste tipo não é viável, dados os custos da sua manutenção. Já a interacção nos laços fracos é menos regular e menos dispendiosa.

Portanto, cada indivíduo pode ter um número muito alargado destes laços, que lhe permitem obter informação única (Granovetter, 1973).

O balanço entre laços fortes e fracos influencia a velocidade, quantidade, qualidade e fiabilidade das trocas de informação e conhecimento (Maskell e Malmberg, 1999), bem como os custos com a sua recolha (Coleman, 1988). Certos autores defendem que redes coesas, onde predominam laços fortes, são mais benéficas, pois tendem a facilitar o desenvolvimento de normas comuns e a partilha de valores, expectativas, identidades e perspectivas, conduzindo ao estabelecimento de obrigações e sanções sociais, confiança, compreensão, reputação e reciprocidade (Granovetter, 1985). Neste sentido, os laços fortes e as redes coesas facilitam o fluxo de informação de elevada qualidade, detalhada e específica (Gulati, 1998; Van Geenhvizen, 2008) e a transferência de conhecimento tácito (Lundvall, 1993) e complexo (Hansen, 1999), sendo particularmente relevantes no acesso a recursos escassos (Lovas e Sorenson, 2008). Os laços fracos e as redes ricas em aberturas estruturais (Burt, 1992) facilitam o acesso a contextos que normalmente não teriam contacto entre si e, portanto, a obtenção de informação nova e variada, que pode ser particularmente importante na iden- 
tificação de novas oportunidades (McEvily e Zaheer, 1999; Low e Abrahamson, 1997). Logo, a rede social de um indivíduo pode determinar a amplitude e a qualidade da informação e conhecimento a que ele tem acesso.

\subsection{A questão da proximidade nas redes sociais}

A literatura das redes sociais considera que a criação de um laço social directo implica pelo menos uma interacção pessoal (face-a-face) entre os dois actores. No entanto, geralmente, as redes sociais envolvem um padrão de relações sociais mais intensas e frequentes entre agentes. Sem esta frequência de contactos aumenta a probabilidade de o laço entre os actores desaparecer. Devido a esta necessidade de interacção, muitos autores defendem a importância da proximidade entre os nós. No entanto, a literatura apresenta, vários conceitos de proximidade relevantes na análise das redes sociais. Por exemplo, Boschma (2005) distingue cinco formas de proximidade: geográfica/espacial, social, cultural/institucional, cognitiva/tecnológica/de conhecimento e organizacional. Estas formas de proximidade são de difícil operacionalização, não sendo sempre clara a fronteira entre elas.

A proximidade geográfica tem merecido a atenção de diversas áreas da literatura económica e social. Com efeito, a par da ideia que os processos de empreendedorismo, de criação de conhecimento e de inovação são colectivos e se encontram inseridos em estruturas sociais, existe uma grande ênfase nas vantagens da co-localização, com vários autores a salientarem a sua importância, quer para o aumento da produtividade e da capacidade de inovação das empresas existentes, quer para a redução das barreiras à entrada de novas empresas. Discute-se, nomeadamente o papel da proximidade geográfica na concentração de actividades, no processo de inovação e na aprendizagem.

Neste contexto, é de salientar o conceito de "economias de aglomeração", suportado na ideia que a co-localização das empresas lhes confere vantagem no acesso a recursos locais, traduzindo-se em reduções de custos associadas a economias de localização e de urbanização. As economias de localização são uma das razões apontadas para a emergência de distritos industriais, consistindo em economias de escala em inputs intermédios, vantagens associadas ao mercado de trabalho e externalidades de conhecimento. As economias de urbanização salientam a importância dos meios urbanos, nomeadamente as suas vantagens em termos de centralização de serviços às empresas ou de serviços públicos (Castells, 1989).

\subsection{Proximidade e transmissão de conhecimento}

Uma das razões apontadas para a aglomeração de empresas é a existência de concentrações regionais/locais de conhecimento, associadas a processos históricos e à importância de factores relacionados com a natureza da base de conhecimento, surgindo distinções como a que separa conhecimento tácito e 
codificado e a que separa conhecimento analítico e sintético (Asheim, 1999; Asheim e Coenen, 2005; Feldman, 1999). Muitos estudos salientam a questão da natureza tácita do conhecimento, reconhecendo um papel importante à colocalização, à mobilidade e aos contactos sociais na transmissão deste tipo de conhecimento. Neste contexto, o conceito de "localized learning" (Maskell et al., 1998) surge associado a vantagens, em termos de criação de conhecimento, da co-localização entre actores envolvidos em actividades relacionadas.

Cabe aqui também uma referência à literatura sobre os spillovers de conhecimento geograficamente concentrados (Jaffe et al., 1993; Audretsch e Feldman, 1996; Autant-Bernard, 2001). O pressuposto base é de que empresas co-localizadas com fontes de conhecimento científico e tecnológico obtêm vantagens, decorrentes da existência de laços sociais e profissionais e de contactos informais mais frequentes entre os investigadores de ambas. O processo de aprendizagem é assim reforçado pela proximidade geográfica com organizações com as quais é possível trocar conhecimento e informação.

$\mathrm{O}$ acesso a recursos humanos qualificados é outra vantagem apontada pela literatura sobre aglomeração. Assim, a existência de universidades facilita o recrutamento em áreas específicas (Wolfe e Gertler, 2001) e a presença de uma massa crítica de quadros qualificados numa região pode favorecer a criação e desenvolvimento de novas empresas.

\subsection{Redes sociais e diferentes formas de proximidade}

Apesar da ênfase colocada na proximidade geográfica, vários autores têm referido que a co-localização entre os actores não é condição suficiente para a ocorrência de processos de transferência de conhecimento (Breschi e Malerba, 2001; Boschma, 2005). Vários estudos revelam que as redes estabelecidas com actores distantes podem ser tão ou mais relevantes do que as estabelecidas com actores próximos e que, frequentemente, as empresas combinam os benefícios de redes que cobrem vários níveis espaciais (Cooke, 2006; Stahecker e Koschatzky, 2004). No caso português, Fontes (2005) analisa as condições em que as novas empresas de biotecnologia obtêm o conhecimento necessário à sua formação e crescimento, concluindo que combinam relações próximas e distantes e, no caso destas últimas, recorrem a vários mecanismos para ultrapassar as desvantagens da distância geográfica no acesso ao conhecimento.

A capacidade de beneficiar de conhecimento existente em organizações geograficamente distantes aponta para a acção das outras formas de proximidade definidas por Boschma (2005). Das cinco formas de proximidade definidas pelo autor, surgem como particularmente relevantes neste trabalho, para além da proximidade geográfica, as proximidades social, cognitiva e organizacional. Com efeito, a investigação centra-se no papel desempenhado pelas redes sociais dos empreendedores e das empresas (o que coloca o ênfase na proximidade social) no acesso ao conhecimento científico e tecnológico (onde a proximidade cognitiva é um requisito), tendo em consideração o processo de construção dessas 
redes, designadamente o percurso dos empreendedores (que é passível de gerar proximidade cognitiva, social e organizacional).

A consideração da proximidade social é uma questão central deste trabalho, já que se recorre à análise de redes sociais para estudar a aglomeração das empresas em torno de centros de investigação. A distância social está relacionada com existência de laços sociais entre os actores ao nível micro, decorrentes da partilha de uma origem ou filiação, ou de determinados atributos sociais (Hausmann, 1996; Sorenson, 2003), sendo de destacar: a facilidade de comunicação, suportada por linguagem e culturas comuns e a confiança, baseada na amizade, parentesco e outros laços decorrentes da experiência pessoal (Casson e Della Giusta, 2007). Desta forma, a proximidade social funciona através da coesão dos actores, facilitando a comunicação entre os membros do grupo ou da rede e, logo, a troca de conhecimento, onde a confiança é essencial (McPherson et al., 2001).

A proximidade cognitiva, associada à partilha de uma base de conhecimento e de competências é igualmente relevante, sobretudo em sectores baseados em tecnologias emergentes como a biotecnologia, devido à natureza frequentemente "exclusiva" e portanto "localizada" do conhecimento a elas subjacente (Antonelli, 1995; Zucker et al., 1998) e à necessidade de combinar conhecimentos de diferentes proveniências (Nooteboom, 2000). Em qualquer caso, a transferência efectiva de conhecimento requer que o receptor tenha capacidade de o absorver (Cohen e Levinthal, 1990), sendo portanto necessário que a estrutura cognitiva deste não seja muito diferente da do emissor. No entanto, para que a troca de conhecimento seja proveitosa, também não deve existir sobreposição total das duas bases de conhecimento.

$\mathrm{Na}$ intersecção entre as proximidades cognitiva e social surgem as "comunidades epistémicas", ou seja, grupos de cientistas, que podem estar mais ou menos dispersos, mas que partilham uma base de conhecimento, uma linguagem e outros códigos de comunicação, e procedimentos de investigação e teste. No seu seio, o conhecimento codificado pode ser considerado um bem público, mas o desconhecimento dos códigos pode levar à exclusão de outros actores (mesmo que co-localizados com o emissor), que não conseguem descodificar as mensagens "abertamente" trocadas (Breschi e Lissoni, 2001).

A proximidade organizacional refere-se à partilha de relações numa base organizacional, estando relacionada com a estrutura de governação hierárquica dessas relações, nomeadamente em termos de autonomia e controlo (Boschma, 2005). Essa estrutura pode ser associada à configuração dos laços da rede social: forte proximidade organizacional traduz-se em laços fortes numa rede hierarquicamente organizada; maior distância organizacional traduz-se em laços fracos entre actores independentes. De acordo com Boschma, quer a proximidade organizacional quer a social estão associadas à existência de laços fortes, embora com diferentes mecanismos (hierarquia e confiança, respectivamente). Neste trabalho surgem associadas às redes sociais dos empreendedores e das empresas e ao seu processo de formação. 


\section{PROXIMIDADE, REDES SOCIAIS E ACESSO AO CONHECIMENTO EM BIOTECNOLOGIA}

Como vimos acima, a literatura realça a importância da proximidade física nos processos de transmissão de conhecimento devido à natureza destes processos. A proximidade física é particularmente relevante quando o conhecimento transmitido tem uma elevada componente tácita (por exemplo, no caso de novas descobertas científicas ou processos em que o "saber fazer" é crítico), quando é de natureza sensível e portanto se coloca a questão da exclusividade, ou quando é complexo ${ }^{4}$.

No entanto, a literatura também sugere que a importância da proximidade física na transmissão do conhecimento científico e tecnológico é reforçada pelo facto de a co-localização ser usualmente determinante para criar outras formas de proximidade, nomeadamente cognitiva, social e organizacional. A proximidade cognitiva é necessária para compreender o conhecimento gerado (nomeadamente quando a sua difusão envolve os códigos próprios de comunidades epistémicas fechadas) e aplicá-lo de forma efectiva. A proximidade social é importante para gerar relações de confiança e facilitar a entrada em comunidades mais exclusivas. Finalmente, a proximidade organizacional (presente ou passada) facilita a interacção porque permite compreender as hierarquias, a organização e os códigos de comportamento das organizações onde o conhecimento é gerado. Como foi argumentado, a simples proximidade física sem proximidade cognitiva, social ou organizacional não garante automaticamente o acesso ao conhecimento, embora permita a criação de condições para o desenvolvimento das outras formas de proximidade, que serão sempre mais difíceis de desenvolver à distância. Com efeito, a interacção frequente face-a-face e a partilha de experiências permitida pela co-localização, geram oportunidades para identificação de interesses comuns e favorecem o desenvolvimento de relações para os explorar conjuntamente, bem como o aprofundamento dessas relações através de experiências múltiplas, permitindo nomeadamente a co-evolução dos actores.

Mas exactamente porque a proximidade física não é condição suficiente, é possível em certas circunstâncias desenvolver proximidade cognitiva, social e organizacional sem existir co-localização continuada. A co-localização temporária pode, em certas condições, substituir-se àquela, designadamente quando o conhecimento a que pretende aceder não se encontra disponível (ou é de mais difícil acesso) no contexto em que o actor se insere. Assim, as redes dos empreendedores podem envolver, quer indivíduos localizados em organizações geograficamente próximas, que tendencialmente serão relações mais fortes, envolvendo interacções frequentes; quer indivíduos localizados em organizações geograficamente mais distantes, que tendencialmente serão relações mais complexas de criar e/ou manter e por essa razão

4 Se o conhecimento é complexo, mesmo a codificação pode não garantir completa compreensão ou reprodução num contexto diferente, sendo necessária a interacção com os seus produtores (Sorenson, 2003). 
estarão associadas ao acesso a conhecimentos científicos e tecnológicos de especial importância ou mais difíceis de aceder no seu contexto geográfico.

A literatura sobre redes pessoais traz um importante contributo para este debate ao possibilitar a análise detalhada do processo de construção dessas redes e, nomeadamente, ao chamar a atenção para o papel dos percursos anteriores dos indivíduos nesse processo. Esses contributos permitem-nos argumentar que embora a construção das redes que facilitam o acesso ao conhecimento científico e tecnológico raramente seja possível sem co-localização, esta pode ter lugar em diferentes momentos do percurso do empreendedor e não ser necessariamente contemporânea do estabelecimento da empresa.

Esta questão assume um relevo particular num contexto de crescente mobilidade inter-organizacional e internacional dos cientistas, nomeadamente nas áreas científicas associadas à biotecnologia (Ackers, 2005). É expectável que relações caracterizadas pela proximidade cognitiva, social, organizacional se vão estabelecendo em localizações diversas, ao longo do percurso do empreendedor e se mantenham quando a co-localização termina (alimentadas pelos avanços das TIC e por novas oportunidades de co-localização temporária) (Saxenian e Hsu, 2001). Tendo em conta que a mobilidade está frequentemente associada à procura de conhecimento mais avançado, também é expectável que os indivíduos e organizações com quem essas relações se estabelecem venham a assumir um papel importante como fontes de conhecimento científico e tecnológico da empresa que vai ser criada.

Assim, as redes que são determinantes para que as novas empresas acedam ao conhecimento científico e tecnológico, podem compreender vários níveis. Podem basear-se na rede pessoal pré-existente dos empreendedores, envolvendo: quem está fisicamente próximo da empresa que se está a constituir; quem esteve fisicamente próximo durante o percurso anterior do empreendedor e, embora já não o esteja, continue a pertencer à sua rede social e seja visto por este como uma fonte importante do conhecimento que a empresa pretende obter. Mas tais redes podem também resultar de um esforço propositado para desenvolver relações de proximidade cognitiva/social/organizacional com quem possui conhecimento considerado relevante para a empresa, independentemente da sua localização. Neste caso, se existirem fontes desse conhecimento na proximidade é expectável que os esforços sejam dirigidos para elas; se não, poderão ser dirigidos para fontes distantes, através da procura de várias instâncias de colocalização temporária. Em ambos os casos o recurso à intermediação da rede social existente (laços indirectos), quando viável, pode ser determinante.

De acordo com a literatura sobre economias de urbanização, será expectável que quanto mais numerosas, diversificadas e qualificadas forem as fontes de conhecimento científico e tecnológico no contexto em que a empresa está localizada, menor seja a necessidade de estabelecer redes distantes, embora possa haver excepções. Por exemplo, se os laços distantes pré-existentes são suficientemente importantes para a empresa, o esforço necessário à sua manutenção pode ser inferior ao necessário à geração de novas relações no local onde a empresa é criada, sobretudo 
se está em causa uma comunidade científica relativamente fechada, ou se não for possível estabelecer redes de confiança com essa comunidade. No entanto, a nível local/regional, poderão não existir fontes de conhecimento científico e tecnológico com as características pretendidas e, nesse caso, haverá uma maior tendência por parte dos empreendedores para recorrer a relações pré-existentes, cuja génese foi a co-localização mas que estão distantes do local onde a empresa é criada. Estas situações podem surgir mesmo em regiões bem providas de fontes de conhecimento, que embora relevantes para empresa, não permitam obter conhecimento particularmente novo ou mais especializado, apenas acessível em outras localizações. Tal significa que as empresas podem ter redes para acesso ao conhecimento com diferentes estruturas geográficas, nas quais exista maior ou menor peso das relações próximas/distantes ou um equilíbrio entre elas (Gittelman, 2007). Estas diferenças na estrutura geográfica das relações podem estar relacionadas com a natureza do conhecimento procurado e a sua importância para a nova empresa, ou com diferenças na natureza das relações.

Relativamente à aglomeração das empresas em torno de centros de produção de conhecimento, é portanto possível concluir que a proximidade geográfica (ou seja a co-localização dos indivíduos) permite gerar relações caracterizadas pela proximidade cognitiva/social/organizacional que facilitam a transmissão do conhecimento científico e tecnológico. A continuidade dessa proximidade geográfica também facilita a manutenção das redes e o seu aprofundamento.

Nesse sentido, a partir das ideias expostas ao longo desta secção, centrando a atenção no acesso ao conhecimento científico e tecnológico, considerando condições idênticas no acesso a outros recursos $\operatorname{críticos}^{5}$ e ignorando factores pessoais dos empreendedores (familiares, profissionais, etc.) é possível argumentar que:

a) existirá uma tendência para os empreendedores localizarem as suas empresas na vizinhança das fontes de conhecimento científico e tecnológico com as quais têm relações mais intensas e que são mais relevantes para a sua actividade;

b) se existirem fontes de conhecimento relevantes geograficamente próximas (e sobretudo se existirem relações de proximidade cognitiva/social/organizacional com os seus cientistas) haverá menos incentivo para mobilizar relações distantes;

c) se tais fontes não existirem localmente, ou se o seu acesso se revelar complexo ou pouco efectivo ${ }^{6}$, os empreendedores tenderão a mobilizar os elementos distantes das redes sociais que foram construídas ao longo do seu percurso.

5 Como já foi referido, o processo de criação de empresas envolve a mobilização de outros recursos para além do conhecimento científico e tecnológico, como o acesso a instalações, a financiamento e a competências complementares ao nível da produção, comercialização, regulação, etc.

6 Por exemplo, de acordo com Michel et al. (2004), num contexto em que existem vários competidores que podem ter acesso ao conhecimento produzido em centros de investigação locais, as empresas podem percepcionar riscos na relação com esses centros (dada a natureza destas organizações "fugas de informação" poderão estar facilitadas) e preferir não se relacionar localmente. 


\section{ABORDAGEM EMPÍRICA AO CASO DAS EMPRESAS PORTUGUESAS DE BIOTECNOLOGIA}

As questões levantadas na secção anterior são investigadas empiricamente para o caso das empresas portuguesas de biotecnologia. Nesta secção apresenta-se brevemente o sector e propõe-se uma metodologia de abordagem que foi aplicada de forma experimental num grupo de 4 empresas, como primeira fase de um processo que irá englobar a totalidade das empresas na área da biologia molecular (22 empresas e 54 empreendedores). Este trabalho exploratório permitiu testar e afinar a metodologia que se apresenta de seguida.

\section{O sector da biotecnologia em Portugal}

O sector da biotecnologia é bastante recente em Portugal. Nos anos 90 criaram-se algumas empresas, mas apenas em meados de 2000 se registou um aumento significativo do número de iniciativas empreendedoras neste domínio. No entanto, o sector é ainda muito incipiente: existem actualmente cerca de 76 "empresas dedicadas à biotecnologia", $80 \%$ das quais criadas nos últimos 5 anos. A maior parte das empresas encontra-se numa fase embrionária e só um pequeno grupo iniciou já a introdução das suas tecnologias/produtos no mercado. As principais áreas de aplicação incluem: saúde tanto humana como animal (43\%), agricultura e produção de alimentos (29\% e $16 \%$ respectivamente) e ambiente $(9 \%)$.

$\mathrm{O}$ incremento verificado nos últimos anos está associado a uma combinação de factores favoráveis (Fontes, 2007). A crescente qualidade e maturidade da investigação em algumas instituições, bem como a presença de elevado número de jovens cientistas altamente qualificados e internacionalizados mas sub-empregados, combinada com mudanças no contexto institucional, conduziram a um aumento dos incentivos a iniciativas empresariais envolvendo a exploração comercial de conhecimento originário da investigação. Este contexto contribui para explicar as características das empresas: a maioria são spin-offs de investigação e parte substancial foi criada por iniciativa de, ou envolvendo, jovens cientistas.

Em termos de localização, as empresas distribuem-se ao longo de uma "faixa litoral", aglomerando-se em torno das principais cidades: particularmente Lisboa (37\%) e Porto (22\%), mas crescentemente Coimbra (12\%) e também Faro $(8 \%)$ e Braga (7\%). Portanto, a maioria encontra-se localizada nas grandes áreas metropolitanas, onde se concentram as principais instituições públicas de investigação e o essencial das infra-estruturas de incubação e de apoio e outros serviços relevantes. A tendência para a localização junto de centros de produção de conhecimento é confirmada se analisarmos com detalhe a posição das empresas face aos principais centros de investigação nacionais nos domínios científicos que contribuem para a biotecnologia. Verifica-se que, não só ao nível de distrito, mas também ao nível de concelho, existe uma forte correlação entre a localização das empresas e a presença desses centros. 
O grupo de 22 empresas da biologia molecular, analisadas neste trabalho, enquadra-se no padrão anteriormente descrito, embora com algumas particularidades. Estas empresas estão aglomeradas em torno de duas cidades: Lisboa (50\%) e Coimbra (27\%). As suas actividades concentram-se no sector da saúde (20 em 22 empresas), com destaque para as aplicações clínicas, que se sobrepõem às farmacêuticas. Todas foram fundadas por empreendedores de origem académica e experiência internacional, embora em alguns casos as equipas promotoras integrem também elementos com perfil não académico. Estas equipas são maioritariamente compostas por jovens empreendedores, ainda que algumas incluam um investigador sénior, que mantém o seu vínculo à universidade ou centro de investigação.

A localização geográfica das empresas estudadas parece apontar para a opção de criar a empresa na proximidade de centros de produção de conhecimento, embora não seja de ignorar que tal localização pode também obedecer a estratégias de acesso a outros recursos, ou a motivos de ordem pessoal. Nesse sentido, é relevante avaliar até que ponto essa opção está de facto associada ao desenvolvimento de relações de natureza científica e tecnológica com os centros de investigação localizados na região, qual a origem dessas relações e ainda qual a sua importância para a empresa, relativamente a outras fontes. É de notar que parte significativa das empresas tem pelo menos um fundador com percurso internacional, frequentemente em centros de excelência internacionais de biotecnologia, o que pode indiciar um papel potencialmente relevante para as redes pessoais estabelecidas com esses centros.

\section{Uma metodologia para análise do papel das redes sociais no acesso ao conhecimento}

Pretende-se contribuir para uma melhor compreensão do processo de aglomeração das empresas de biotecnologia em regiões onde se localizam os principais centros de produção de conhecimento, avaliando o papel desempenhado pelas redes mobilizadas com esses núcleos no acesso ao conhecimento, comparativamente com o desempenhado por redes eventualmente estabelecidas com fontes mais distantes.

Na condução desta análise tem-se em consideração: i) que o conhecimento científico e tecnológico é um recurso crítico para este tipo de empresas; ii) que a proximidade geográfica tem um papel importante no acesso ao conhecimento, embora não seja suficiente para garantir esse acesso, devendo existir igualmente proximidade cognitiva, social, organizacional; iii) o papel das redes sociais construídas pelos empreendedores ao longo do seu percurso académico e profissional no desenvolvimento de proximidade cognitiva, social e organizacional. Com base nesses pressupostos, procura-se compreender:

a) até que ponto as decisões de localização da empresa tomadas pelos empreendedores são influenciadas pela proximidade geográfica em relação a 
centros de produção de conhecimento e, nomeadamente pela variedade e qualidade dos centros existentes numa dada região;

$b$ ) até que ponto as redes sociais construídas pelos empreendedores ao longo do percurso influenciam estas decisões, quer reforçando a tendência para localizar a empresa próximo de centros com os quais existem relações-chave; quer permitindo a localização da empresa em locais mais ou menos distantes geograficamente de centros com os quais existem relações-chave, mas onde se verifiquem outro tipo de vantagens.

Para tratar este problema desenvolveu-se uma abordagem metodológica em dois passos. O primeiro envolve a (re)construção das redes sociais que são mobilizadas no acesso ao conhecimento científico e tecnológico durante o processo de criação e desenvolvimento inicial das empresas, com vista a compreender a sua composição e a forma como foram constituídas ${ }^{7}$. O segundo envolve uma análise de proximidade relativamente aos centros de produção de conhecimento, com vista a compreender a importância da aglomeração em torno desses centros e o papel relativo de outras formas de proximidade no acesso ao conhecimento científico e tecnológico. São consideradas três unidades de análise: a) a natureza das redes sociais das empresas; b) a proximidade entre as empresas e os centros de produção de conhecimento; c) a natureza do contexto local/regional em termos de presença, diversidade e qualidade de centros de investigação ${ }^{8}$.

\subsection{A (re)construção das redes das empresas}

Como foi referido, o percurso profissional e académico dos empreendedores é relevante na formação das redes sociais das empresas, sobretudo na fase inicial, onde a rede da empresa se confunde com a do empreendedor (Hsu, 2007). Por outro lado, durante o processo de constituição e na fase inicial da empresa, são estabelecidas novas relações (formais, ou formalizadas a partir de certo ponto) que têm já como objecto a empresa.

A informação sobre as redes mobilizadas no acesso ao conhecimento científico e tecnológico é obtida combinando fontes documentais (CVs, publicações, patentes, projectos) com informação recolhida em entrevistas com os empreendedores, baseadas em questionários semi-estruturados ${ }^{9}$.

7 A análise recai sobre o processo de constituição e a fase inicial da empresa, que se considerou englobar o ano de criação e os dois anos seguintes. Numa fase posterior da investigação considerar-se-á também a situação actual.

8 Numa fase posterior do trabalho será interessante considerar outros elementos de caracterização da região como o nível de desenvolvimento e factores institucionais e políticos locais/regionais que afectam o processo de formação de empresas.

9 Esta metodologia conjuga métodos aplicados individualmente por vários autores (Casper, 2007; Breschi e Lissoni, 2004; Murray, 2004;). Para uma descrição detalhada ver Sousa et al., (2008). 
As entrevistas permitem identificar os elementos da rede pessoal que foram efectivamente mobilizados pela empresa no acesso ao conhecimento científico e tecnológico, bem como as redes intencionalmente criadas com esse objectivo ao nível da empresa. Uma vez que o intuito deste trabalho é analisar a relação entre empresas e centros de produção de conhecimento, optou-se pela consideração do nível organizacional, identificando-se, para cada indivíduo da rede social do empreendedor, a organização (ou organizações) a que se encontra ligado.

A rede social da empresa resulta da junção das redes de percurso mobilizadas e das redes intencionais. Na sua construção considera-se a intensidade dos laços existentes com cada membro. Assim, as redes são construídas de forma a repercutirem o número de relações entre os empreendedores/empresa e cada fonte de conhecimento. Contabiliza-se também o número de recursos obtidos através de cada relação, o que permite equacionar a intensidade com que os empreendedores recorrem à rede social para obter os recursos de que necessitam.

As redes assim obtidas permitem identificar as fontes de conhecimento da empresa na sua fase inicial, ter uma primeira aproximação à importância relativa de cada uma delas e compreender onde se encontram localizadas. Uma análise mais fina permitirá ainda avaliar até que ponto as empresas recorrem a diferentes fontes para aceder a outros tipos de recursos.

A titulo ilustrativo da aplicação desta metodologia de (re)construção de redes apresenta-se, na figura 1, a representação gráfica da rede social mobilizada para acesso ao conhecimento científico e tecnológico, no período inicial da actividade de uma das empresas estudadas na fase experimenta ${ }^{10}$. Os membros da rede são representados por círculos, se forem centros de investigação, e por quadrados, se forem outro tipo de organização. A distância geográfica é visível na cor do símbolo: branco se a empresa e a outra organização estiverem localizadas na mesma região, cinzenta se estiverem no mesmo país e preta se estiverem em países distintos. Do ponto de vista gráfico, a intensidade dos laços é representada pela espessura do traço que liga os actores.

A observação desta figura permite ilustrar algumas das questões equacionadas ao longo deste trabalho. Constata-se que a rede de acesso a conhecimento científico e tecnológico é composta por organizações localizadas em diferentes níveis espaciais. Neste caso, em termos numéricos predominam os centros de investigação internacionais, mas as ligações mais intensas (traço mais espesso) são mantidas com organizações de investigação locais (actores 18 e 78) e nacionais (actor 19). Uma análise mais detalhada da relação mais intensa (estabelecida com o actor 18) revela que, para além da proximidade física, ela é marcada por outras formas de proximidade, já que este actor se encontra presente nos percursos dos empreendedores. Globalmente, a análise aprofundada da origem e

10 Utilizou-se o software UCINET (versão 6 para Windows) para construir a rede e computar as medidas de intensidade e distância e o software NetDraw para produzir a respectiva representação gráfica (Borgatti, 2002 e Borgatti et al., 2002). 
natureza dos laços aqui representados, bem como dos respectivos conteúdos, permitirá avaliar o nível e o(s) tipo(s) de contributo de cada uma das organizações e, ainda, aferir uma eventual associação com a sua localização ou com a forma como os seus membros foram angariados.

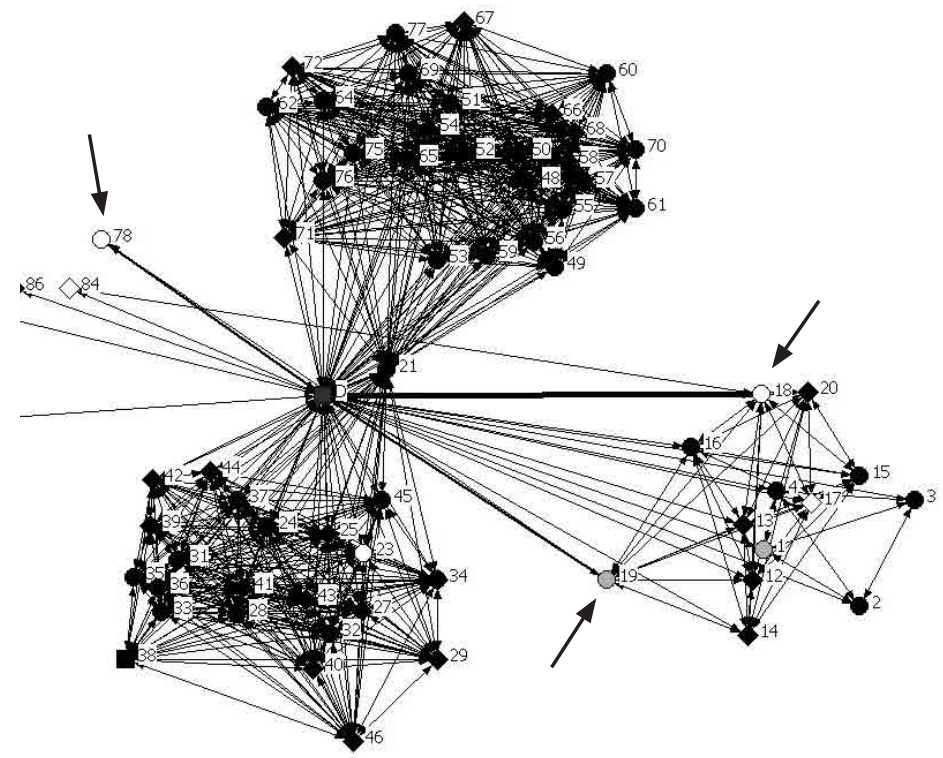

Fig. 1 - Uma rede social de acesso a conhecimento.

Fig. 1 - A knowledge social network.

\subsection{Análise da proximidade e da natureza do contexto local/regional}

Relativamente à proximidade geográfica, a literatura apresenta várias medidas. Neste caso, optou-se por definir níveis espaciais, acompanhando o reconhecimento crescente na literatura de que os clusters locais estão inseridos numa matriz geográfica mais vasta (Bunnell e Coe, 2001). Assim, definiu-se a seguinte escala de proximidade entre as empresas de biotecnologia e os centros de investigação: co-localização (localizados na mesma morada, sendo a distância nula); local (concelho), regional (distrito), nacional (país) e internacional ${ }^{11}$.

A proximidade social é caracterizada usando medidas das redes sociais (Wasserman e Faust, 1994), considerando-se neste caso três medidas de distância social: a distância máxima, a distância média e a centralidade de proximidade (closeness centrality).

11 Numa fase posterior poder-se-á combinar esta escala com medidas de distância em quilómetros ou em horas de viagem. Adicionalmente, poder-se-á analisar a dimensão espacial a que ocorrem os fenómenos de proximidade no acesso a conhecimento científico e tecnológico, de acordo com a sugestão avançada por um referee, cuja contribuição se agradece. 
A proximidade cognitiva é obtida recorrendo a duas proxies: a primeira resulta do cruzamento entre a(s) área(s) de actividade(s) da empresa e as áreas de investigação do centro ${ }^{12}$; a segunda reflecte a participação da empresa (ou do empreendedor) e do centro de investigação em projectos conjuntos. Desta forma, procura-se capturar a semelhança das bases de conhecimento e a partilha de códigos de comunicação e de procedimentos de investigação e teste entre a empresa e o centro de investigação.

Quanto à proximidade organizacional parte-se da ideia de que, se o empreendedor "passou" pelo centro produtor de conhecimento durante o seu percurso, tem algum conhecimento sobre os valores, estruturas hierárquicas, normas e rotinas desse centro. A medida utilizada neste caso será a existência (ou não) de uma ligação prévia do empreendedor ao centro durante o seu percurso académico ou profissional.

Para caracterizar o contexto local/regional procede-se à identificação, para cada empresa, dos centros de investigação que se encontram co-localizados (mesma morada), localizados no mesmo concelho e localizados no mesmo distrito. Neste levantamento foram consideradas as Unidades de I\&D do Programa de Financiamento Plurianual da Fundação para a Ciência e Tecnologia (FCT) e os "Laboratórios Associados", nos domínios científicos que contribuem para a biotecnologia, tendo sido identificados $131 \operatorname{centros}^{13}$. Com base na informação sobre a localização de cada centro e de cada empresa é construída uma matriz que permite contabilizar quantos centros estão na mesma morada, no mesmo concelho e no mesmo distrito que cada uma das empresas e, assim, caracterizar o contexto local/regional em termos do número de centros de produção de conhecimento. São ainda introduzidas três medidas que permitem qualificar esse contexto: dimensão - baseada no número de investigadores de cada centro; variedade - baseada nas áreas de especialização dos centros; qualidade - baseada na classificação obtida na avaliação externa conduzida pela FCT.

\section{CONCLUSÕES}

Neste artigo aborda-se a aglomeração de novas empresas de biotecnologia em torno de núcleos de produção de conhecimento científico e tecnológico, visando compreender o papel da mobilização de redes locais no acesso ao conhecimento, comparativamente com o papel desempenhado por redes estabelecidas com fontes mais distantes.

12 Numa fase posterior poder-se-á obter uma medida mais precisa, recorrendo a peritos para comparar a um nível mais fino as áreas de conhecimento definidas pelas empresas com as áreas de especialização dos centros.

13 A este grupo adicionou-se ainda um centro criado recentemente no domínio da biotecnologia (Biocant), que não se enquadra naqueles grupos mas desempenha um papel de catalisador de novas empresas na Região Centro. 
O caso das novas empresas de biotecnologia em Portugal - enquanto exemplo de contexto onde existe uma base de conhecimento razoavelmente desenvolvida e se verifica um número crescente de iniciativas empreendedoras neste domínio, embora esteja longe de se poder considerar um importante cluster de biotecnologia - suscita algumas questões, sobre as estratégias de localização e sua relação com as estratégias de acesso ao conhecimento. Essas questões estão também reflectidas na literatura sobre este sector, que ora aponta para uma tendência para a aglomeração à volta dos principais centros de conhecimento e negócio, realçando o papel da proximidade geográfica, ora chama a atenção para o facto de as empresas tenderem a desenvolver um leque extenso de relações geograficamente distantes.

A abordagem apresentada neste artigo, combinando a literatura sobre redes sociais com debates recentes sobre proximidade, possibilita ultrapassar uma abordagem linear baseada na dicotomia "proximidade-distância". Assim, a análise das redes mobilizadas no acesso ao conhecimento científico e tecnológico - quer as redes pessoais dos empreendedores, quer as redes intencionalmente criadas pela empresa - permite, antes de mais, avaliar a utilização de fontes geograficamente próximas e, portanto, em princípio "disponíveis" e "acessíveis", bem como o papel de fontes geograficamente mais distantes. Por outro lado, a literatura de redes - através da consideração da influência dos percursos dos empreendedores na formação da sua rede social - e a literatura sobre proximidade - realçando o papel da proximidade social, cognitiva e organizacional - oferecem-nos uma perspectiva teórica para abordar as estratégias de aquisição de conhecimento que se afastam da simples lógica da proximidade física.

Com base nessas contribuições define-se um conjunto de proposições, que se podem sumarizar da seguinte forma: a) as empresas procurarão localizar-se na vizinhança de fontes de conhecimento relevantes para a sua actividade, embora a decisão final de localização seja também determinada por uma combinação de outros motivos; b) se existirem fontes relevantes geograficamente próximas (e sobretudo se existirem relações de proximidade cognitiva/social/organizacional com os seus cientistas) haverá menos incentivo para mobilizar relações distantes; c) se tais fontes não existirem, ou se o seu acesso se revelar complexo, ou se verificar que fontes distantes são mais efectivas (ou mesmo únicas), os empreendedores tenderão a mobilizar para esse fim os elementos distantes das redes sociais que foram construídas ao longo do seu percurso.

Para explorar empiricamente esta abordagem, foi desenvolvida uma metodologia que permite: a) reconstruir as redes mobilizadas no acesso ao conhecimento científico e tecnológico, tendo em vista compreender a sua composição, origem e estrutura; b) desenvolver uma primeira aproximação à medição das várias formas de proximidade (geográfica, social, cognitiva, organizacional) das empresas em relação às principais fontes de conhecimento. Esta metodologia foi afinada através da aplicação experimental num pequeno grupo de casos e encontra-se actualmente em implementação num universo mais amplo das empresas de biotecnologia portuguesas. 
Os resultados desta investigação irão permitir uma melhor compreensão da forma como as empresas de biotecnologia acedem a conhecimento científico e tecnológico num país de desenvolvimento intermédio e do papel desempenhado por diversas formas de proximidade nesse processo de acesso ao conhecimento. Tal permitirá, nomeadamente, apoiar a formulação de políticas públicas, quer as que favorecem a formação de empresas spin-offs em torno núcleos de produção de conhecimento, quer políticas apoiando as estratégias empresariais de acesso a conhecimento distante ou, numa fase anterior, à mobilidade como elemento base para a formação de redes mobilizáveis para o acesso ao conhecimento.

\section{BIBLIOGRAFIA}

Ackers L (2005) Moving people and knowledge: the mobility of scientists within the European Union. International Migration, 43: 99-129.

Aldrich H E, Zimmer C (1986) Entrepreneurship through social networks. In Smilor R, Sexton D (eds.) The art and science of entrepreneurship. Ballinger, Cambridge: 154-167.

Antonelli C (1995) The economics of localized technological change and industrial dynamics. Kluwer, Dordrecht.

Asheim B T (1999) Interactive learning and localized knowledge in globalizing learning economies. GeoJournal, 49(4): 345-352.

Asheim B T, Coenen L (2005) Knowledge bases and regional innovation systems: comparing Nordic clusters. Research Policy, 34: 1173-1190.

Audretsch D B, Feldman M (1996) Spillovers and the geography of innovation and production. American Economic Review, 86: 630-640.

Audretsch D B, Stephan P E (1996) Company-scientist locational links: the case of biotechnology. American Economic Review, 86 (3): 641-652.

Autant-Bernard C (2001) The geography of knowledge spillovers and technological proximity. Economics of Innovation and New Technology, 10: 237-254.

Bönte W (2008) Inter-firm trust in buyer-supplier relations: are knowledge spillovers and geographical proximity relevant? Journal of Economic Behaviour \& Organization, 67: 855-870.

Borgatti S P (2002) Netdraw network visualization. Analytic Technologies, Harvard MA.

Borgatti S P, Everett M G e Freeman L C (2002) Ucinet for windows: software for social network analysis. Analytic Technologies, Harvard MA.

Boschma R A (2005) Proximity and innovation: a critical assessment. Regional Studies, 39: 61-74.

Breschi S, Lissoni F (2004) Knowledge networks from patent data: methodological issues and research targets. Cespri Working Paper $\mathrm{N}^{\circ} 150$.

Breschi S, Lissoni F (2001) Knowledge spillovers and local innovation systems: a critical survey. Industrial and Corporate Change, 10: 975- 1005.

Breschi S, Malerba F (2001) The geography of innovation and economic clustering: some introductory notes. Industrial and Corporate Change, 10: 817-833.

Bunnell T, Coe N (2001) Spaces and scales of innovation. Progress in Human Geography, 25: 569-589. 
Burt R (1992) Structural holes: the social structure of competition. Harvard University Press, London.

Carsrud A, Johnson R (1989) Entrepreneurship: a social psychological perspective. Entrepreneurship \& Regional Development, 1: 21-31.

Casper S (2007) How do technology clusters emerge and become sustainable? Social network formation and inter-firm mobility within the San Diego biotechnology cluster. Research policy, 36: 438-455.

Casson M, Della Giusta M (2007) Entrepreneurship and social capital - analysing the impact of social networks on entrepreneurial activity from a rational action perspective. International Small Business Journal, 25(3): 220-244.

Castells M (1989) The informational city. information technology, economic restructuring, and the urban-regional process. Basil Blackwell, Oxford.

Castilla E, Hwang H, Granovetter E, Granovetter M (2000) Social networks in Silicon Valley. In Lee C M, Miller W, Hancock M G, Rowen H R (eds.) The Silicon Valley edge - a habitat for innovation and entrepreneurship. Stanford University Press, Stanford: 217-247.

Cohen W M, Levinthal D A (1990) Absorptive capacity: a new perspective on learning and innovation. Administrative Science Quarterly, 35: 128-152.

Coleman J (1988) Social capital in the creation of human capital. American Journal of Sociology, 94: $95-120$.

Cooke P (2006) Global bioregional networks: a new economic geography of bioscientific knowledge. European Planning Studies, 14(9): 1265-1285.

Ensley M D, Hmieleski K M (2005). A comparative study of new venture top management team composition, dynamics and performance between university-based and independent start-ups. Research Policy, 34(7): 1091-1105.

Feldman M (1999) The new economics of innovation, spillovers and agglomeration: a review of empirical studies. Economics of Innovation and New Technology, 8: 5-25.

Fontes M (2007) Technological entrepreneurship and capability building in biotechnology. Technology Analysis and Strategic Management, 13(3): 351-367.

Fontes M (2005) Distant networking: the knowledge acquisition strategies of 'out-cluster' biotechnology firms. European Planning Studies, 13(6): 899-920.

Fornahl D (2005) The impact of regional social networks on the entrepreneurial development process. In Fornahl D, Zellner C, Audretsch D A (eds.) The role of labour mobility and informal networks for knowledge transfer. Springer, Bloomington: 53-78.

Gittelman M (2007) Does geography matter for science-based firms? Epistemic communities and the geography of research and patenting in biotechnology. Organization Science, 18(4): 724-741.

Granovetter M (1985) Economic action and social structure: The problem of embeddedness. American Journal of Sociology, 91: 481-510.

Granovetter M (1973) The strength of weak ties. American Journal of Sociology, 78: 1360-1380.

Gulati R (1998) Alliances and networks. Strategic Management Journal, 19: 293-317.

Hansen M T (1999) The search-tranfer problem: the role of weak ties in sharing knowledge across organization studies. Administrative Science Quarterly 44: 82-111.

Hausmann U (1996) Neither industrial district nor innovation milieu: entrapreneurs and their contexts. an actor-oriented framework and case studies from Greater London and Zurich. European Regional Science Association 36th European Congress, Zurich. 
Hsu D (2007) Experienced entrepreneurial founders, organizational capital, and venture capital funding. Research Policy, 36: 722-741.

Jaffe A B, Trajtenberg M, Henderson R (1993) Geographic localization and knowledge spillovers as evidenced by patent citations. Quarterly Journal of Economics, 108: 577-598.

Johannisson B (1998) Personal networks in emerging knowledge-based firms: spatial and functional patterns. Entrepreneurship \& Regional Development, 10: 297-312.

Kogut B, Zander U (1992) Knowledge of the firm, combinative capabilities, and the replication of technology. Organization Science, 3: 383-397.

Lane P, Lubatkin M (1998) Relative absorptive capacity and interorganizational learning. Strategic Management Journal, 19 (5): 461-477.

Liebeskind J P, Oliver A L, Zucker L, Brewer M (1996) Social networks, learning and flexibility: sourcing scientific knowledge in new biotechnology firms. Organization Science, 7:428- 443.

Lovas B, Sorenson O (2008) The mobilization of scarce resources. In Baum J A, Rowley T J (eds.) Advances in strategic management: network strategy.Vol. 25. JAI Press, Amsterdam: 361-389.

Low M B, Abrahamson E (1997) Movements, bandwagons and clones: industry evolution and the entrepreneurial process. Journal of Business Venturing, 12: 435- 457.

Lundvall B A (1993) Explaining interfirm cooperation and innovation. Limits of the transaction-cost approach. In Grabher G (ed.) The embedded firm. On the socioeconomics of industrial networks. Routledge, London: 52-64.

Maskell P, Malmberg A (1999) Localised learning and industrial competitiveness. Cambridge Journal of Economics, 23: 167-185.

Maskell P, Eskelinen H, Hannibalson I, Malmberg A, Vatne E (1998) Competitiveness, localized learning and regional development: specialization and prosperity in small open economies. Routledge, London.

McEvily B, Zaheer A (1999) Bridging ties: a source of firm heterogeneity in competitive capabilities. Strategic Management Journal, 20: 1133-1156.

McPherson M, Smith-Lovin L, Cook J (2001) Birds of a feather: homophily in social networks. Annual Review of Sociology, 27: 415-444.

Michel J, Quére M, Selosse S (2004) Life Sciences/Biotechnology Study in the Provence-Alpes-Cote d'Azur (Paca) Region, Report Project Tell SERD-2000-00115, http://www.fondazionerosselli. it/User.it/index.php?PAGE=Sito_it/centri_ricer1\&unit_id=14\&rice_id=227 [Acedido a $12 \mathrm{de}$ Janeiro de 2009].

Murray F (2004) The role of inventors in knowledge transfer: sharing in the laboratory life. Research Policy, 33: 643-659.

Nooteboom B (2000) Learning and innovation in organizations and economies. Oxford University Press, Oxford.

Owen-Smith J, Powell W W (2004) Knowledge networks as channels and conduits: the effects of spillovers in the boston biotechnology. Organization Science, 15(1): 6-21.

Pisano G P (1991) The governance of innovation: Vertical integration and collaborative arrangements in the biotechnology industry. Research Policy, 20: 237-249.

Powell W, Koput K W, Bowie J I, Smith-Doerr L (2002) The spatial clustering of science and capital: accounting for biotech firm-venture capital relationships. Regional Studies, 36(3): 291-305. 
Powell W, Koput K, Smith-Doerr L (1996) Interorganizational collaboration and the locus of innovation: networks of learning in biotecnology. Administrative Science Quarterly, 41: 116-145.

Saxenian A, Hsu J Y (2001) The silicon Valley-Hsinchu connection: technical communities and industrial upgrading. Industrial and Corporate Change, 10: 893-920.

Shane S, Venkataraman S (2000) The promise of entrepreneurship as a field of research. Academy of Management Review, 25: 217-226.

Sorenson O (2003) Social networks and industrial geography. Journal of Evolutionary Economics, 13: $513-527$.

Sousa C, Fontes M, Videira P (2008) The role of entrepreneurs' social networks in the creation and early development of biotechnology companies. RENT XXI Conference, Covilhã, 20-21 November 2008.

Stahlecker T, Koschatzky K (2004) On the significance of geographical proximity for the structure and development of newly founded knowledge-intensive business service firms. Working Papers Firms and Region, No. R2/2004, Fraunhofer Institute.

Stuart T, Sorenson O (2003) The geography of opportunity: spatial heterogeneity in founding rates and the performance of biotechnology firms. Research Policy, 32: 229-253.

Uzzi B (1997) Social structure and competition in interfirm networks: the paradox of embeddedness. Administrative Science Quarterly, 42: 35-67.

Van Geenhuizen M (2008) Knowledge networks of young innovators in the urban economy: biotechnology as a case study. Entrepreneurship \& Regional Development, 20: 161-183.

Wasserman S, Faust K (1994) Social network analysis: methods and applications. Cambridge University Press, Cambridge.

Witt U, Zellner C (2007) Knowledge-based entrepreneurship: the organizational side of technology commercialization. In Malerba F, Brusoni S (eds.) Perspectives on innovation. Cambridge University Press, Cambridge: 352-371.

Wolfe D A, Gertler M S (2001) Globalization and economic restructuring in Ontario: from industrial heartland to learning region?.European Planning Studies, 9(5): 575-592.

Zucker L, Darby M, Brewer M (1998) Intelectual capital and the birth of US biotechnology enterprises. American Economic Review, 88: 290-306. 